\title{
Three Dimensional In Vitro Culture of Murine Secondary Follicles in a Defined Synthetic Matrix
}

\author{
Mohammad Asaduzzman ${ }^{1 *}$, Xiaolin $\mathrm{Cui}^{2}$, Hu Zhang2, Fiona Young1 \\ ${ }^{1}$ Medical Biotechnology, Flinders University, Adelaide, Australia \\ ${ }^{2}$ School of Chemical Engineering, The University of Adelaide, Adelaide, Australia \\ Email: *asad0009@flinders.edu.au, steven.cui@otago.ac.nz, hu.zhang@adelaide.edu.au, fiona.young@flinders.edu.au
}

How to cite this paper: Asaduzzman, M., Cui, X.L., Zhang, H. and Young, F. (2018) Three Dimensional In Vitro Culture of Murine Secondary Follicles in a Defined Synthetic Matrix. Journal of Biomaterials and Nanobiotechnology, 9, 244-262. https://doi.org/10.4236/jbnb.2018.93014

Received: March 26, 2018

Accepted: June 29, 2018

Published: July 4, 2018

Copyright (c) 2018 by authors and Scientific Research Publishing Inc. This work is licensed under the Creative Commons Attribution International License (CC BY 4.0).

http://creativecommons.org/licenses/by/4.0/

\begin{abstract}
Ovarian follicle growth in three dimensional (3D) matrices in vitro has limitations: a) matrices don't expand as follicles grow, b) requirements for enzyme-mediated retrieval, and c) animal-derived components prevent clinical application. Therefore, we evaluated N-Isopropylacrylamide (SFX-1), a novel synthetic 3D culture matrix, for follicle culture. Groups of three murine secondary follicles were encapsulated in $50 \mu \mathrm{L}$ of DMEM/F12-1\%ITS-10\%FCS (DMEM/F12) or SFX-1 (3:2 v/v DMEM/F12) or Matrigel (1:1 DMEM/F12) and cultured for $48 \mathrm{~h}$. Matrigel contains growth factors but SFX-1 has no animal-derived factors. Each culture condition was examined in 6 wells containing 18 follicles, in four replicate experiments $(n=4)$. Photomicrographs were used to determine follicle diameters and morphological integrity. Follicles were Live-Dead (LD) stained or disaggregated to generate cells for viability assessment using Trypan Blue (TB). Estradiol, progesterone and anti-mullerian hormone (AMH) in conditioned media were measured using Enzyme-linked Immunoassay. All culture conditions supported similar increases in follicle diameter. DMEM/F12 did not maintain morphological integrity which prevented follicle retrieval after $48 \mathrm{~h} ; 25 \%$ were retrieved from DMEM/F12, but $44 \%$ and $41 \%$ follicles were retrieved from SFX-1 and Matrigel respectively. Follicles retrieved from Matrigel could not be disaggregated, which prevented TB viability assessment. $\mathrm{LD}$ estimations of viable cells/follicle were lower than $\mathrm{TB}$, but culture conditions had no effect on viability; SFX-1 64\% $\pm 8 \%$ and DMEM/F12 69\% $\pm 9 \%$. SFX-1 and Matrigel supported similar levels of progesterone synthesis, only Matrigel supported estrogen synthesis, but none of the culture conditions supported AMH production. SFX-1 was not cytotoxic and was comparable to Matrigel. Further development of SFX-1 for use with human follicles is supported.
\end{abstract}




\section{Keywords}

Ovarian Follicle, Synthetic Matrix, Matrigel, Estradiol, Progesterone, Anti-Mullerian Hormone

\section{Introduction}

Ovaries contain follicles which are three dimensional (3D) spheres made up of a central oocyte (egg) surrounded by layers of granulosa cells, and additional layers of theca cells in the larger, more mature follicles. The main function of the granulosa and theca cells is to support oocyte maturation so that it can be fertilized by spermatozoa after ovulation in vivo [1]. Ovarian follicles are cultured in vitro for many reasons including in vitro maturation to produce fertilizable oocytes, and to allow investigation of the fertility-suppressing effects of chemotherapeutics on granulosa cell viability and hormone production [2]-[9]. Two-dimensional (2D) culture systems have been used to grow follicles but these cause collapse of the 3D spherical structure with consequent impairment of follicle and oocyte maturation [5] [10] [11] [7]. In contrast, culturing ovarian follicles in 3D matrices supports the growth of follicles in all directions, maintains inter-cellular gap junctions and maintains enclosure of the oocyte within the granulosa cell layers [5] [7]. The purpose of this study is to compare two 3D matrices (established versus novel) with a $2 \mathrm{D}$ follicle culture system (negative control).

Two dimensional (2D) culture systems allow cells to grow in a monolayer on a rigid and flat substrate [12]. 2D follicle culture systems have been evaluated by examining oocyte germinal vesicle break-down (GVB) [10] [13] and meiotic arrest [10], both indicators of oocyte maturation and fertilisability, as well as follicle granulosa cell viability [8], follicular growth to antral stage [8] [14], follicular integrity [15] [16] [17], and the production of steroid hormones; estrogen (E2) and progesterone (P4) [8] [7]. However, the application of 2D follicle culture systems to the in vitro maturation of follicles and oocytes has been criticized because they do not maintain follicular integrity throughout the culture period, and granulosa cells tend to grow out through the follicle basal lamina within four days of culture [7]. Moreover, 2D culture systems do not model the in vivo 3D environment in which follicles are enclosed by the extracellular matrix of the ovary [12]. Follicular integrity is essential for follicle and oocyte maturation because granulosa cells communicate constantly with each other and the oocyte through gap junctions [5] [15] [16] [17]. When follicles are cultured in 2D systems, stress on the intercellular gap junctions causes a significant reduction in oocyte growth, extrusion of oocytes, loss of spherical structure [5] [7] [10] [11] [7] and flattening of the follicles [18]. Although follicles cultured in 2D systems grow and can exhibit 2.1 to 5.3 -fold increases in diameter [10] [11], only $62 \%$ of the oocytes undergo GVB [13] and the oocyte diameters tend to be significantly smaller after 10 days in culture than those of freshly isolated oocytes from fol- 
licles of comparable age [10] [11]. It was argued that the enzymatic disaggregation of ovarian tissue to collect follicles damaged the basal laminae of the isolated follicles and that this compromised follicular integrity [5], contributed to the detachment of granulosa cells from each other and the oocyte [7] and impaired maturation. However, when follicles were collected mechanically from the ovaries without enzymes, cultured in a 2D system for 6 days and the viability assessed using Live-Dead stain, the follicular viability was only $8 \%$ [19]. In some studies, follicles were said to be viable if the follicle retained a 3D spherical structure and granulosa cells enclosed the oocyte [8], but in situ studies indicated that about $20 \%$ to $40 \%$ of granulosa cells in a follicle can be non-viable, irrespective of species [20]-[26] and in isolated morphologically intact, spherical follicles $10 \%$ of granulosa cells were not viable when measured by staining the whole follicle with Live-Dead stain [27] or by Trypan Blue staining [28]. Together, these studies indicate that $2 \mathrm{D}$ culture systems are not optimal for follicular and oocyte maturation.

Three dimensional (3D) in vitro culture matrices have been developed to overcome the limitations of $2 \mathrm{D}$ culture systems. In vivo, the distinct cellular compartments of ovarian follicles; theca cells, basal laminae, granulosa cells and the cumulus oocyte complex (COC) [5], are embedded in an extracellular matrix consisting of collagen, fibrinogen and hyaluronic acid [18] [29] [30] [31] [32]. The follicles are in turn embedded in the extracellular matrix of the ovary which consists of collagen, proteoglycans, and tenascin C [33] [34]. There are two broad types of 3D matrices; alginate and Matrigel. Alginate matrices are derived from algae and comprise $\beta$-d-mannuronic acid and $\alpha$-l-guluronic acid that forms gel by ionic cross-linking of the guluronic residue. Higher concentrations of alginate $(0.25 \%)$ however reduced the growth and maturation of follicles and oocytes [35] [36] [37] but incorporation of components of extra cellular matrix such as collagen I and IV, fibronectin and laminin into alginate increased steroid hormone ( $\mathrm{P} 4$ and $\mathrm{E} 2$ ) production by follicles, probably because it promoted the adhesion of granulosa cells to each other and the oocyte [20] [38] [39]. Fibrin-alginate based matrix is responsive to follicle growth because proteases present in the matrix are activated by follicular cells during growth [40]. Matrigel is an extract from tumor cells and consists of collagen IV, fibronectin, laminin, entactin, sulfate proteoglycans, heparins and growth factors [41]. Liquid Matrigel forms a gel at or above $20^{\circ} \mathrm{C}$ and hence when cultured at $37^{\circ} \mathrm{C}$, supports follicular growth and integrity [41]. Matrigel however, does not allow expansion or growth of follicles over time [5]. Another problem associated with alginate and Matrigel 3D matrices is the need to use enzymes to retrieve follicles when the culture is finished [5] [20] [40].

Three dimensional culture systems must allow gas exchange and diffusion of nutrients, and this permeation is affected by the porosity of the matrix. 3D culture matrices must be not toxic to the growing cells and ideally should also allow sustainable growth for the duration of the culture period (Desai et al 2010) by supporting the growth of follicles in all directions and by holding the cellular 
and non-cellular compartments close together within a spherical structure [18]. Another factor affecting in vitro follicle culture is the co-culture of groups of follicles together, because this has been reported to promote oocyte development and follicular growth irrespective of other aspects of the culture system [14] [18] possibly because follicles can communicate with each other, as in the in vivo situation.

Human follicles are collected from various patient cohorts, primarily to culture and produce fertilizable oocytes that can be submitted to established clinical IVF protocols which will enable infertile couples to have children. Regulatory agencies do not permit human cells that will be implanted or transferred into patients to be exposed to animal-derived products [42]. Many advances have been made to optimize in vitro culture systems for follicles, but at the time of writing no method has been unequivocally proven to be effective for consistent follicular development in vitro. The animal-derived Matrigel culture system holds promise but needs modification to allow non-destructive isolation of the follicle from the gel, and further data are required to determine if the fibrin-alginate interpenetrating network (FA-IPN) follicle culture system supports correct morphological development.

Healthy premenopausal human ovarian tissue is scarce, and few human follicles are available for research and development. Therefore, the objective of this study was to evaluate SFX-1, a novel 3D culture matrix, in terms of its ability to support murine 3D follicular growth, and to determine if the new culture matrix is worth further study using human follicles in the future. SFX-1 is a thermosensitive synthetic matrix that is fully defined and has no components derived from animals, hence it has potential for clinical application for ovarian follicles, and also for the culture of other 3D mammalian structures in vitro. The SFX-1 matrix is in solution status below $33^{\circ} \mathrm{C}$ and gels above this temperature. This property suggested it might be easier to encapsulate and retrieve follicles from SFX-1 than from Matrigel. On the other hand, SFX-1 does not contain proteins, growth factors or structural components whereas Matrigel does, and this led to the hypothesis that follicles cultured in DMEM/F12 with $10 \%$ FCS in a $2 \mathrm{D}$ system would flatten, lose their spherical structure and fail to produce hormones, whereas follicles cultured in SFX-1 with DMEM/F12 and 10\% FCS would retain their spherical structure and synthesize hormones, and follicles cultured in Matrigel with DMEM/F12 and 10\% FCS would have the best morphology and highest hormone production. Since this is the first study to examine follicle culture in SFX-1, it was important to determine if the new matrix was immediately and acutely cytotoxic, therefore the study aims were to quantify murine follicle cell viability, follicular growth, morphological integrity (maintenance of 3D spherical structure), and hormone production in a short 48-hour culture in vitro.

\section{Materials and Methods}

All reagents from Sigma Aldrich (Australia) unless otherwise stated. 


\subsection{Synthesis of SFX-1}

The SFX-1 matrix was synthesized by free radical emulsion polymerization as reported previously [43]. Before the polymerization, the monomer N-Isopropylacrylamide (NIPAM) was recrystallized in n-hexane and dried in vacuum at room temperature, and acrylic acid (AA) was purified by vacuum distillation. For the SFX-1 synthesis, $9.9 \mathrm{mmol}$ NIPAM, $0.1 \mathrm{mmol} \mathrm{AA,} 0.2 \mathrm{mmol} \mathrm{N}$, $\mathrm{N}$ '-methylenebis (acrylamide) and $0.12 \mathrm{mmol}$ of sodium dodecyl sulphate were added in $97 \mathrm{~mL}$ of water. The solution was bubbled with nitrogen for 30 minutes. $3 \mathrm{~mL}$ of potassium persulphate aqueous solution $(0.1 \mathrm{mmol})$ was then injected into the degassing solution to initiate the polymerization at $70^{\circ} \mathrm{C}$. After polymerization for $5 \mathrm{~h}$ with continuous supply of nitrogen and stirring, the temperature was reduced to the ambient temperature. The as-prepared products were purified by membrane dialysis with a cut-off molecular weight of $12-14 \mathrm{kDa}$ against Milli-Q water for one week with a daily water change.

\subsection{Preparation of Reagents}

Dulbecco's Modified Eagle's Medium with Hams F12 was supplemented with $1 \%$ insulin $(5 \mathrm{ug} / \mathrm{mL})$, transferrin $(5 \mathrm{ug} / \mathrm{mL})$ and selenium $(5 \mathrm{ng} / \mathrm{mL}$, "ITS", Gibco, New York, USA), and $1 \%$ penicillin $(10,000$ units $/ \mathrm{mL})$ and streptomycin $(10 \mathrm{mg} / \mathrm{mL})$ and hereafter referred to as DMEM/F12. For use in all three culture systems heat inactivated foetal calf serum (FCS, DKSH, Melbourne, AUS) was added to DMEM/F12 to $10 \% \mathrm{v} / \mathrm{v}$.

SFX-1 $(50 \mathrm{mg} / \mathrm{mL})$ was diluted to $30 \mathrm{mg} / \mathrm{mL}$ with $40 \% \mathrm{v} / \mathrm{v}$ DMEM/F12 and $10 \%$ FCS on the day before each experiment. Matrigel $(8.61 \mathrm{mg} / \mathrm{mL})$ was diluted 1:1 with DMEM/F12 and 10\% FCS immediately before encapsulating follicles. Live-Dead combined stain containing $10 \mu \mathrm{M}$ Calcein AM and $20 \mu \mathrm{M}$ Ethidium Homodimer-1 (Invitrogen, Paisley, UK) was prepared in $1 \mathrm{X}$ phosphate buffered saline (PBS). Collagenase IV (12 mg/mL, 295 units/mg, Worthington, New Jersey, USA) was prepared in DMEM/F12 and hyaluronidase $(0.025 \%, 1228$ units/mg, Lakewood, NJ) was prepared in 1X PBS.

\subsection{Mouse Ovary Collection}

Mature adult female mice (C57 BL, $10.7 \pm 1$ weeks, $\mathrm{n}=8$ ) were killed by cervical dislocation and the isolated ovaries placed in $37^{\circ} \mathrm{C}$ DMEM/F12 and transported to the laboratory at $37^{\circ} \mathrm{C}$. These mice were excess to the needs of the breeding colony and allocated to routine cull. They were euthanized by College of Medicine animal facility staff, and the use of their ovaries for this research project was approved by the Animal Welfare Committee at Flinders University, Adelaide, South Australia.

\subsection{Follicle Isolation from Ovaries}

Whole ovaries were dissected free of oviducts, adipose tissue, and extraneous tissue, cut in half, and disaggregated using $0.5 \mathrm{~mL}$ collagenase IV $(2 \mathrm{mg} / \mathrm{mL}$ in 
DMEM/F12) for 30 minutes at $37^{\circ} \mathrm{C}$ with 1 minute agitation after every $10 \mathrm{mi}$ nutes, then mechanically disaggregated with 22-gauge syringe needles for $5 \mathrm{mi}$ nutes. All released follicles were collected and transferred to a 96-well plate containing DMEM/F12 with 10\% FCS.

\subsection{Follicle Size Classification}

Follicle diameters were initially estimated using a scale graticule in the lens eye piece of a dissecting microscope at $3 \mathrm{X}$ magnification. Follicles were separated into pools of primordial, primary, early secondary, late secondary or antral follicles according to the size ranges described by Griffin J, Emery BR, Huang I, Peterson CM and Carrell DT [44] and Young J and McNeilly AS [45]. The follicles were collected from two mice on four separate occasions $(n=4$ experimental replicates). On each occasion, all the secondary follicles from two mice were combined into one pool of follicles and examined using a dissection microscope to identify follicles with excellent morphology (see below for scoring system). These were then randomly distributed to culture in SFX-1, Matrigel or DMEM/F12, such that at the beginning of culture, all follicles had high M1 or M2 scores for morphology and spherical integrity.

\subsection{Follicle Culture}

$20 \mu \mathrm{L}$ of Matrigel or SFX-1 or DMEM/F12 with 10\% FCS were added to wells of a 96 well plate and incubated at $37^{\circ} \mathrm{C}$ in a humidified incubator for 5 minutes. Groups of three secondary follicles were added to each well, and overlain with 30 $\mu \mathrm{L}$ of Matrigel or SFX-1 or DMEM/F12. These were incubated at $37^{\circ} \mathrm{C}$ for another 5 - 10 minutes, to ensure that follicles were encapsulated within each matrix. The solid hydrogels (each well containing three follicles) were then overlain with $100 \mu \mathrm{L}$ DMEM/F12 with $10 \%$ FCS, and the follicles cultured for 48 $\mathrm{h}$ at $37^{\circ} \mathrm{C}$ with $5 \% \mathrm{CO}_{2}$. The SFX-1 gel contained $40 \%$ and the Matrigel contained 50\% DMEM/F12 with 10\% FCS, whereas the follicles in the 2D culture system were surrounded by 100\% DMEM/F12 with FCS. Each of the three culture systems (Matrigel, SFX-1 and DMEM/F12) were examined in 6 replicate wells, so a total of 18 follicles were used to examine each culture condition within each of 4 experimental replicates. Follicles were photographed using an inverted microscope immediately after encapsulation and before culture. After 48 hours at $37^{\circ} \mathrm{C}$, media were collected, and the follicles were left at room temperature for ten minutes to allow the matrices to cool and become liquid gel. This allowed the follicles to be retrieved without enzymes and washed with PBS. Nine follicles from 3 replicate wells were subjected to Live-Dead staining whereas follicles from the other three replicate wells were disaggregated (see below) and the isolated granulosa cells assessed using a Trypan Blue exclusion assay.

\subsection{Live-Dead Staining}

Follicles $(n=27)$ in each replicate experiment $(n=4)$ were washed with PBS 
then stained with $150 \mu \mathrm{L}$ Live-Dead combined stain at $37^{\circ} \mathrm{C}$ for 45 minutes. The follicles were washed with PBS before fixation in $150 \mu \mathrm{L}$ of $4 \%$ paraformaldehyde (PFA) for 40 minutes in the dark at room temperature. The Live-Dead stained whole follicles were placed onto a poly-L-lysine coated glass microscope slide with $5 \mu \mathrm{L}$ buffered glycerol and a cover slip.

Images of Live-Dead stained whole follicles were captured using a fluorescence microscope (Brightfield BX50, Olympus) at 20x magnification using Micro-Manager (v1.4.13) image capture software. The fluorescence microphotographs were analysed using Image J $(1.49 \mathrm{v})$, and the diameters of each follicle were determined by drawing a straight line across the follicle three times then calculating the average.

After subtracting non-specific background fluorescence, the grey scale image was used to identify all Live-stained, or Dead-stained areas. Overlapping stained areas were subdivided by applying an Image J plugin program "Watershed". A "Particle analyser" function in Image J was then used to count the number of separate stained areas. These were outlined to check against the original image, and the estimated numbers were recorded for each follicle.

\subsection{Follicle Disaggregation and Trypan Blue Exclusion Assay}

Follicles $(n=27)$ in each replicate experiment $(n=4)$ were disaggregated in collagenase IV for 30 minutes before treatment in hyaluronidase for another 30 minutes. The granulosa cells harvested from each group of 3 disaggregated follicles were resuspended in $20 \mu \mathrm{L}$ DMEM/F12, then $20 \mu \mathrm{L}$ Trypan Blue was added to these cell suspensions. $10 \mu \mathrm{L}$ was loaded onto a haemocytometer, and the live and dead cells in 9 large squares were counted. The mean \pm SD numbers of viable or dead granulosa cells were expressed per follicle, and the numbers of viable cells were expressed as percentage of the total number of granulosa cells from one follicle.

\subsection{Follicle Growth and Morphology}

After $48 \mathrm{~h}$ culture, only the diameters of the Live-Dead stained follicles were measured because follicles from the other three technical replicates were disaggregated and the granulosa cells were assessed using Trypan Blue. The morphology of each Live-Dead stained follicle was classified as being intact (M1, the follicle appeared completely enclosed by the basal lamina), or largely intact but of lower morphological integrity (M2, the basal lamina appeared irregular or broken in places, but without any loss or displacement of GC), or slightly (M3) or completely disrupted (M4) [46] [47].

\subsection{Progesterone Measurement}

In each experiment and for each culture condition (SFX-1 or Matrigel or DMEM/F12), conditioned media were collected from two replicate wells, each containing three follicles, after 48 hours of culture. The conditioned medium 
from each culture well was examined in one well of a competitive enzyme-linked immunoassay (EIA, Cayman Chemical ELISA, Ann Arbor, MI, USA), and duplicate wells were used to measure progesterone production. A mouse monoclonal anti-rabbit IgG, and an acetylcholinesterase progesterone tracer were used for the progesterone EIA. The manufacturer reported a detection limit of $10 \mathrm{pg} / \mathrm{mL}$, an intra-assay coefficient of variation (CV) of 7.5\%, and an inter-assay CV of $2.9 \%$ for this assay. For this study, the progesterone standard was diluted in DMEM/F12 with 10\% FCS to give concentrations that ranged from 7.18 to 1000 $\mathrm{pg} / \mathrm{mL}$

\subsection{Estradiol Measurement}

In each experiment, and for each culture condition, conditioned media were collected from two replicate wells (each containing 3 follicles) after 48 hours of culture and examined in a competitive EIA (Cayman Chemical ELISA, Ann Arbor, MI, USA) that used a mouse anti-rabbit IgG, and an acetylcholinesterase estradiol tracer. The manufacturer reported a detection limit of $15 \mathrm{pg} / \mathrm{mL}$, and an intra-assay coefficient of variation (CV) of $7.8 \%$ to $18.8 \%$ for this assay. For this study, the estradiol standard was diluted in the DMEM/F12 with $10 \%$ FCS to give concentrations that ranged from 6.6 to $4000 \mathrm{pg} / \mathrm{mL}$.

\subsection{Anti-Mullerian Hormone (AMH) Enzyme Immunoassay}

As described above, conditioned media from two replicate wells (each containing 3 follicles) were collected after 48 hours of culture. Conditioned media samples were examined in a two-step sandwich type EIA (Immunotech, Marseille Cedex, France) that used an anti-AMH monoclonal antibody for capturing AMH, and biotinylated monoclonal antibody together with streptavidin-peroxidase for detecting bound $\mathrm{AMH}$ in the wells. The manufacturer reported a detection limit of $1 \mathrm{pg} / \mathrm{mL}$, an intra-assay coefficient of variation (CV) of $12 \%$, and an inter-assay $\mathrm{CV}$ of $14.2 \%$. For this study, the AMH standards were diluted in DMEM/F12 with $10 \%$ FCS to give concentrations that ranged from 0 to $150 \mathrm{pM}$.

\subsection{Statistical Analysis}

The mean \pm SD values of four experimental replicate $(n=4)$ were determined for each culture condition and experimental output (follicle diameter, granulosa cell viability, P4, E2 or AMH concentration). Follicle diameters were subjected to one-way ANOVA with Tukey's Multiple Comparison test, whereas the numbers of live and dead granulosa cells were subjected to a two-way ANOVA with Bonferroni post-test. Statistical analyses were carried out using GraphPad Prism, and statistical significance was assigned at $\mathrm{p}<0.05$.

\section{Results}

\subsection{Follicle Growth}

The initial diameter of the subset of secondary follicles that were allocated to 
Live-Dead staining was the same as the entire cohort of secondary follicles (Table 1 ), and there was no difference between the follicles that were randomly allocated to each of the three culture conditions (SFX-1, Matrigel or DMEM/F12) at the beginning of the experiment (Table 1$)$. There was a significant increase $(\mathrm{p}<$ $0.05)$ in follicle diameter in all three matrices after 48-hour culture, but no difference between the three culture conditions; the SFX-1 matrix supported follicle growth to the same extent as Matrigel.

\subsection{Follicle Morphology}

Green Live-staining was localised to the cytoplasm of granulosa cells, although in some follicles the staining was more punctate (Figure 1(A)) than in others (Figure 1(B)). Diffusive Live-staining was seen in the vicinity of the oocyte. Red Dead-staining was localised to the nuclei of granulosa cells (Figure $1(\mathrm{C})$ ) and to the large nucleus of the oocyte in highly disrupted follicles with low M4 morphology scores (Figure 1(D)). Fewer than half the follicles were retrieved from the wells (SFX 44\%, Matrigel 41\%, DMEM/F12 25\% retrieved) because in many cases the granulosa cells attached to the floor or wall of the culture wells and formed a monolayer, and the oocytes were not enclosed by granulosa cells (Figure 1(E)).

All the follicles ( $\mathrm{n}=36$ in each matrix) had an intact spherical morphology which was scored as being M1 or M2 at the commencement of culture. The highest numbers of follicles were retrieved (and Live-Dead stained) from SFX-1 after $48 \mathrm{~h}$ culture ( $\mathrm{n}=16$ follicles), a similar number $(\mathrm{n}=15)$ were retrieved after culture in Matrigel, but only nine follicles were retrieved after $48 \mathrm{~h}$ culture in the 2D DMEM/F12 system. The follicles that could not be retrieved from the culture wells did not have an intact spherical structure and were more disrupted than the follicles given M4 scores (Figure 1(E)), hence the highest number of follicles with poor, non-spherical morphology resulted from culture in DMEM/F12.

Matrigel maintained an intact spherical M1 or M2-graded morphology in 47\% of the follicles that could be retrieved (Figure 2), the 2D DMEM/F12 culture system did not support the maintenance of any follicles with M1 scores, although

Table 1. Follicle Growth after $48 \mathrm{~h}$ in vitro.

\begin{tabular}{ccccc}
\hline & $\begin{array}{c}\text { Initial Follicle } \\
\text { Diameter } \\
(\mu \mathrm{m})\end{array}$ & $\begin{array}{c}\text { Initial Diameter }(\mu \mathrm{m}) \text { of } \\
\text { Follicles allocated to } \\
\text { Live-Dead staining }\end{array}$ & $\begin{array}{c}\text { Final Diameter } \\
(\mu \mathrm{m}) \text { of Live-Dead } \\
\text { stained follicles }\end{array}$ & $\begin{array}{c}\text { Percent } \\
\text { increase }(\%)\end{array}$ \\
\hline SFX-1 (3D) & $144 \pm 20$ & $153 \pm 28$ & $201 \pm 38^{*}$ & $43 \pm 30$ \\
Matrigel (3D) & $152 \pm 8$ & $152 \pm 5$ & $213 \pm 42^{*}$ & $40 \pm 29$ \\
DMEM/F12 (2D) & $148 \pm 6$ & $145 \pm 6$ & $205 \pm 22^{*}$ & $41 \pm 18$ \\
\hline
\end{tabular}

Murine secondary follicles $(n=216)$ were cultured in groups of three for $48 \mathrm{~h}$ in SFX-1, Matrigel or DMEM/F12. Half the follicles were assessed using Live-Dead stain. Photomicrographs of follicles were taken before and after culture, and the images used to determine diameter. The mean \pm SD diameter of follicles in 4 replicate experiments shown $(\mathrm{n}=4)$, and the data assessed by one-way ANOVA with Tukey's Multiple Comparison test. ${ }^{*} \mathrm{p}<0.05$. 

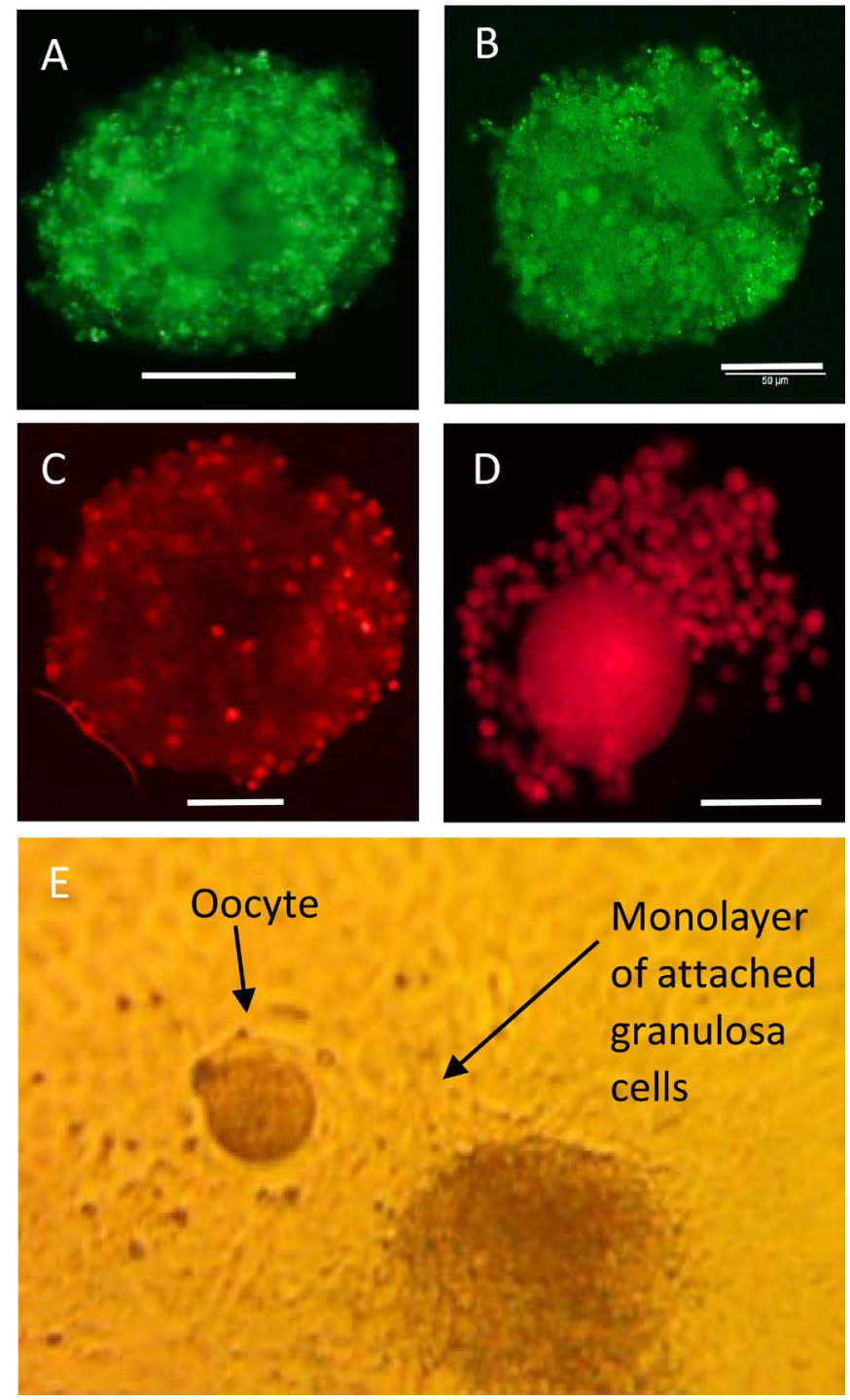

Figure 1. Effect of Culture on Follicle Viability and Morphology. Intact spherical murine secondary follicles with high morphological quality scores of M1 and M2 were cultured for $48 \mathrm{~h}$ in 3D or 2D matrices, retrieved and Live-Dead stained. (A) An M2 Live-stained follicle cultured in Matrigel. (B) M3 Live-stained follicle cultured in SFX-1. (C) M3 Dead-stained follicle cultured in SFX-1. (D) Disrupted M4 Dead-stained follicle cultured in DMEM/F12. (E) Disrupted follicle with extruded oocyte in a culture well that could not be retrieved nor Live-Dead stained.

$44 \%$ of the low number of follicles that were retrieved had M2 scores, and in SFX-1 only $12.5 \%$ of retrieved follicles maintained M1 or M2 morphological scores. There were however significantly higher numbers of follicles with M3 scores $(\mathrm{p}<0.01)$ in SFX-1 than those with M1 or M2 scores.

\subsection{Follicular Cell Viability}

Although follicles were washed free of Matrigel, it was not possible to produce a single cell suspension that could be assessed in a Trypan Blue Exclusion assay. The numbers of cells in follicles grown in 3D SFX-1 were not significantly higher 


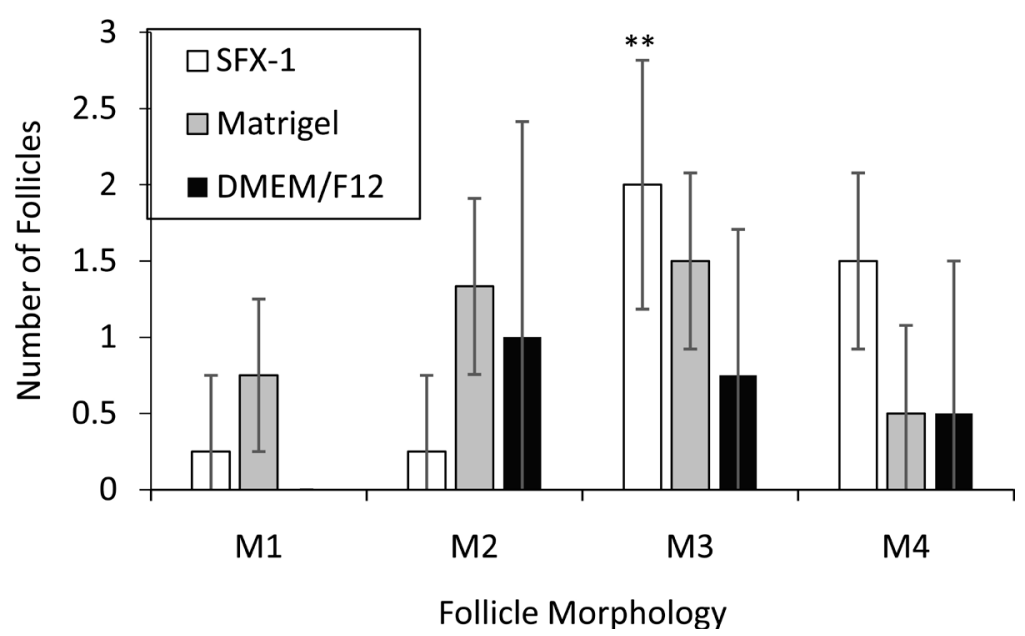

Figure 2. Effect of Culture on Follicle Morphology. Intact spherical murine secondary follicles with high morphological quality scores of M1 and M2 were cultured for $48 \mathrm{~h}$ in 3D SFX-1 $(n=36)$ or Matrigel $(n=36)$, or in 2D DMEM/F12 $(n=36)$. The follicles were retrieved, Live-Dead stained and given scores for spherical integrity and morphological quality. M1 and M2 no loss of granulosa cells, M3 < 10\% loss of granulosa cells, M4 highly disrupted with $>10 \%$ loss of granulosa cells. The experiment was repeated on four separate occasions ( $\mathrm{n}=4,9$ follicles per matrix per experiment) and Mean \pm StDev number of follicles in each morphological category shown. SFX-1 data subjected to 1-Way ANOVA with Tukey post-test, ${ }^{* *} \mathrm{p}<0.01$ compared to M1 \& M2.

than in follicles grown in 2D DMEM/F12 cell culture medium (Figure 3(A)) when assessed by Trypan Blue, and the percentage of viable cells was similar; 64 \pm 8 and $69 \pm 9$ in SFX-1 and DMEM/F12 respectively. Intact Live-Dead stained follicles were assessed using an Image Analysis protocol, which generated data suggesting that these follicles contained more granulosa cells (average $486 \pm 49$, Figure 3(B)) than the disaggregated follicles assessed by Trypan Blue (average $279 \pm 108$, Figure $3(\mathrm{~A})$ ). The percentages of viable cells in intact Live-Dead stained follicles were similar after culture in the three matrices; $8 \% \pm 10 \%$ in SFX-1, $20 \% \pm 6 \%$ in Matrigel and $14 \% \pm 13 \%$ in DMEM/F12 (Figure 3(B)).

\subsection{Follicle Hormone Production}

After a $48 \mathrm{~h}$ culture period, follicles cultured in SFX-1, Matrigel and DMEM did not produce any detectable AMH (Table 2). Follicles cultured in SFX-1 and Matrigel produced progesterone, and only follicles that were cultured in Matrigel produced estrogen.

\section{Discussion}

Mouse ovarian follicles were cultured in a novel, fully defined 3D synthetic gel system for the first time and compared with follicles grown in Matrigel or DMEM/F12 for a short 48h period. Although the only nutrients in the SFX-1 3D gel system derived from the medium (DMEM/F12 with 10\% FCS and 1\% ITS), the diameters and morphological integrity of follicles grown in SFX-1 were not significantly lower than those grown in Matrigel and were clearly superior to 

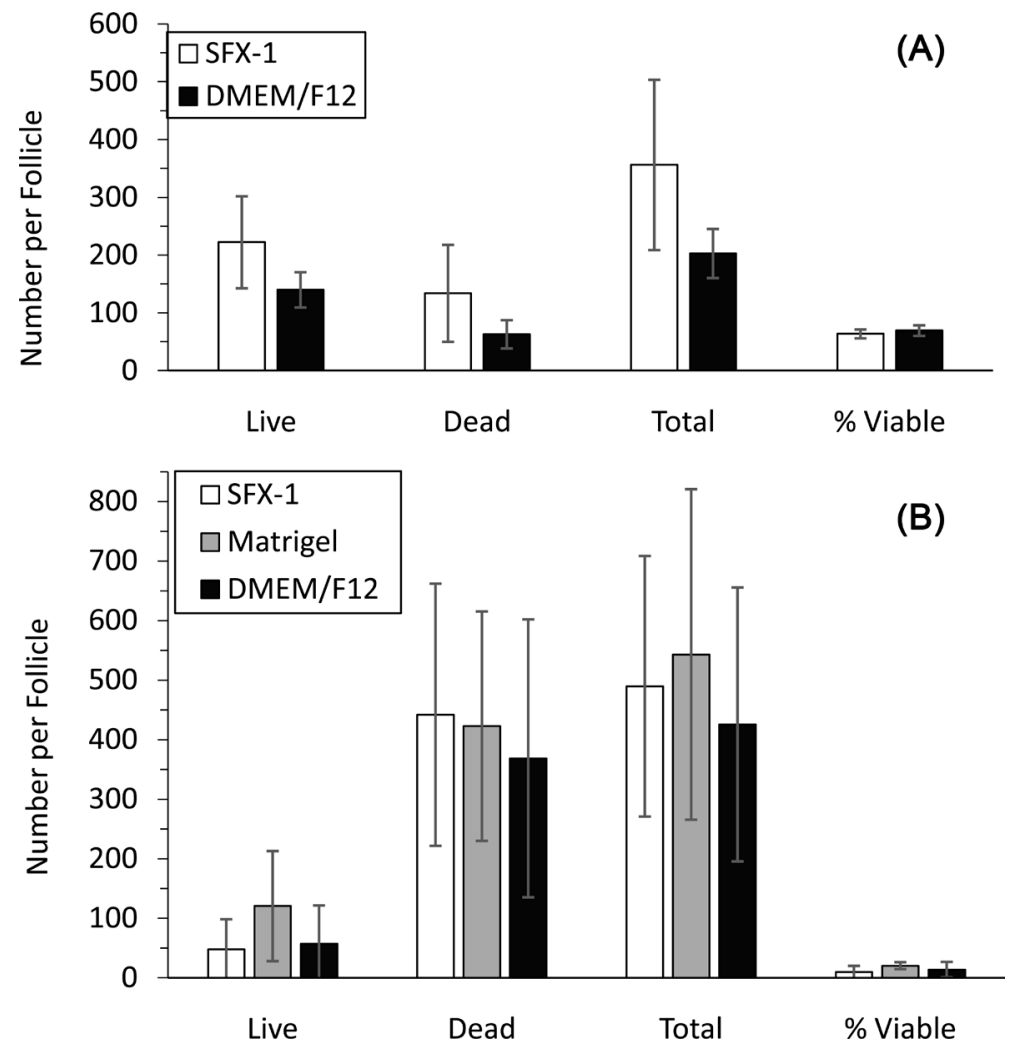

Figure 3. Effect of $48 \mathrm{~h}$ Follicle Culture on Cell Viability. Groups of three murine secondary follicles per well were cultured in SFX-1, Matrigel or in DMEM/F12 medium. After $48 \mathrm{~h}$ in vitro, follicles were (A) disaggregated to produce a single cell suspension which was assessed using a Trypan Blue Exclusion assay to identify live and dead cells or (B) Live-Dead stained and Image Analysis used to quantify the number of areas of staining. Live cells were expressed as a percentage of total cells per follicle. The experiment was repeated on four separate occasions $(n=4)$ and Mean \pm StDev number per follicle shown.

Table 2. Hormone produced by follicles after 48 hours culture.

\begin{tabular}{cccc}
\hline Hormone & SFX-1 & Matrigel & DMEM/F12 \\
\hline AMH & ND & ND & ND \\
E2 & ND & $17.8 \pm 9$ & ND \\
P4 & $478 \pm 285$ & $506 \pm 210$ & ND
\end{tabular}

Groups of three follicles were cultured in SFX-1, Matrigel or DMEM/F12 for $48 \mathrm{~h}$. Conditioned media were collected, and anti-mullerian hormone (AMH), estradiol $17 \beta(\mathrm{E} 2)$ and progesterone (P4) produced by groups of 3 follicles were measured in duplicate by enzyme immuno-assay. The mean $\pm S D \mathrm{pg} / \mathrm{mL}(\mathrm{n}=4)$ shown. ND: not detectable.

those grown in DMEM/F12 with 10\% FCS. All three culture systems supported short-term growth as indicated by increases in follicle diameters, but the morphological integrity scores were highest in Matrigel, as expected. This was probably because Matrigel is a biologically active matrix that contains collagen, laminin and sulfate proteoglycans, [30] as well as other growth factors such as EGF, FGF, IGF-1, PDGF and TGF- $\beta$ [41], and these facilitated adhesion of granulosa cells to each other and the oocyte [20]. Nevertheless, it was clear that 3D structural support in the presence of minimal nutrients, as characterized by the SFX-1 
culture system, was sufficient to support follicle growth and maintain a spherical structure. In addition to this, the highest numbers of follicles were retrieved from SFX-1. Although more than half of the encapsulated follicles in the 3D systems could not be retrieved, a problem noted by others who used 3D matrices containing collagen [5] [48] [49], in future studies components of the ovarian ECM [18] [29] [30] [31] [32] may be incorporated into SFX-1 in a gradual process designed to identify the combination that maximizes follicle growth and retrieval.

The total numbers of granulosa cells estimated by Live-Dead staining were higher than those estimated by Trypan Blue. This discrepancy was probably a consequence of the heterogeneous Live-staining localization which confounded our automated Image Analysis protocol; many areas of Live-staining were smaller than the area of a granulosa cell, and hence the total number of granulosa cells in Live-stained follicles appeared higher than that obtained using the Trypan Blue exclusion assay. Nevertheless, both protocols yielded values similar to those in published reports; 100-400 granulosa cells per secondary follicle [44] [50] [51].

There were concerns that the enzyme disaggregation of follicles, and the centrifugation force applied to retrieve the disaggregated granulosa cells, might have reduced viability before assessment in the Trypan Blue exclusion assay [25] [26] [30] [52] [53], but the proportions of viable granulosa cells were lower when quantified using Live-Dead stain ( 15\%) than when using Trypan Blue ( 65\%). The Live-Dead stain identification of viable cells is based on activity of cytoplasmic esterase enzyme and conversion of Calcein AM to fluorescent Calcein. However, in vitro follicle culture at atmospheric oxygen levels and $5 \% \mathrm{CO}_{2}$ is associated with increased production of Reactive Oxygen Species which in turn reduce the activity of cytoplasmic esterase [19] [54] [55] [56]. Trypan Blue measures cell membrane integrity while Live-Dead stain measures enzyme activity, and the different mechanisms of action may account for the differences in viable cell quantification found in this study. Irrespective of the method used to quantify viable cells, fewer than half of the cells appeared to be viable in each follicle after $48 \mathrm{~h}$ in vitro, whereas in situ studies reported that $80 \%$ of granulosa cells were viable [20] [21] [22] [23] [24]. It was surprising that Matrigel did not maintain significantly higher viable cell numbers than either of the other two culture systems. Altogether our results suggest that the isolation of follicles from ovarian tissue, followed by a $48 \mathrm{~h}$ culture at atmospheric oxygen levels and 5\% $\mathrm{CO}_{2}$ in any system, reduced the viability of follicular granulosa cells. In future, modified SFX-1 will be used in conjunction with other factors that promote follicle growth in vitro, such as the partial pressures of oxygen and $\mathrm{CO}_{2}$ [19] [37] [57] [58].

Hormones produced by granulosa cells are an important indicator of follicle viability and maturation. Matrigel supported the production of both estrogen and progesterone but none of the culture systems supported AMH production, 
even though it has been reported that secondary follicles express the gene for AMH [59] [60] [61] [62]. It is possible than the relatively low levels of granulosa cell viability, and impaired cell-cell communication [5], may have prevented AMH synthesis and secretion.

Our hypothesis was supported; Matrigel supported the growth of high quality morphologically intact follicles that produced steroid hormones, but the retrieval of follicles from Matrigel was difficult. Follicles cultured in DMEM/F12 with $10 \%$ FCS in a $2 \mathrm{D}$ system lost their spherical structure and failed to produce hormones, in contrast to follicles also cultured in DMEM/F12 and 10\% FCS but with the addition of SFX-1, which retained their spherical structure and synthesized the steroid hormone progesterone. Importantly, SFX-1 was not acutely cytotoxic to follicular cells during $48 \mathrm{~h}$ in vitro.

\section{Conclusion}

We conclude that SFX-1 is a promising synthetic 3D culture matrix that was not cytotoxic and supported follicle growth, in the absence of any animal-derived structural components or growth factors, apart from those in 10\% FCS. This study supports further development of SFX-1 for clinical application to the in vitro maturation of follicles, and we recommend future studies in which defined extracellular components and recombinant growth factors are added to SFX-1 for the extended culture of follicles.

\section{Acknowledgements}

We appreciate the financial support awarded to us by Flinders Fertility Pty Ltd., which supported some of this research with a grant managed by the University. The company had no oversight or influence on the research or results.

\section{References}

[1] Macchiarelli, G., Vizza, E., Nottola, S.A., Familiari, G. and Motta, P.M. (1992) Cellular and Microvascular Changes of the Ovarian Follicle during Folliculogenesis: A Scanning Electron Microscopic Study. Archives of Histology and Cytology, 55, 191-204. https://doi.org/10.1679/aohc.55.Suppl_191

[2] Picton, H.M., Harris, S.E., Muruvi, W. and Chambers, E.L. (2008) The In Vitro Growth and Maturation of Follicles. Reproduction, 136, 703-715. https://doi.org/10.1530/REP-08-0290

[3] Zhang, M., Su, Y.Q., Sugiura, K., Wigglesworth, K., Xia, G. and Eppig, J.J. (2011) Estradiol Promotes and Maintains Cumulus Cell Expression of Natriuretic Peptide Receptor 2 (NPR2) and Meiotic Arrest in Mouse Oocytes in Vitro. Endocrinology, 152, 4377-4385. https://doi.org/10.1210/en.2011-1118

[4] Mohammadi-Rousheh, A., Noori-Mooghahi, M., Pasbakhsh, P., Abddvahabi, A., Akbari, M. and Sobhani, A.S.A. (2006) Effect of Cysteamine on the Rate of In Vitro Maturation of Oocytes in Two Media. Acta Medica Iranica, 44, 167-171.

[5] Desai, N., Alex, A., AbdelHafez, F., Calabro, A., Goldfarb, J., Fleischman, A. and Falcone, T. (2010) Three-Dimensional In Vitro Follicle Growth: Overview of Culture Models, Biomaterials, Design Parameters and Future Directions. Reproductive 
Biology and Endocrinology, 8, 119. https://doi.org/10.1186/1477-7827-8-119

[6] Moon, Y., Tsang, B., Simpson, C. and Armstrong, D. (1978) 17 $\beta$-Estradiol Biosynthesis in Cultured Granulosa and Thecal Cells of Human Ovarian Follicles: Stimulation by Follicle-Stimulating Hormone. The Journal of Clinical Endocrinology \& Metabolism, 47, 263-267. https://doi.org/10.1210/jcem-47-2-263

[7] Cortvrindt, R., Smitz, J. and Van Steirteghem, A. (1996) Ovary and Ovulation: In-Vitro Maturation, Fertilization and Embryo Development of Immature Oocytes from Early Preantral Follicles from Prepuberal Mice in a Simplified Culture System. Human Reproduction, 11, 2656-2666. https://doi.org/10.1093/oxfordjournals.humrep.a019188

[8] Heidari, M., Malekshah, A., Azami, N. and Mirhoseini, S. (2012) In Vitro Maturation of Mouse Preantral Follicles in Two Culture Media: A Comparative Study. Annals of Biological Research, 3, 4937-4941.

[9] Telfer, E.E. and Zelinski, M.B. (2013) Ovarian Follicle Culture: Advances and Challenges for Human and Nonhuman Primates. Fertility and Sterility, 99, 1523-1533. https://doi.org/10.1016/j.fertnstert.2013.03.043

[10] Eppig, J.J. and Schroeder, A.C. (1989) Capacity of Mouse Oocytes from Preantral Follicles to Undergo Embryogenesis and Development to Live Young after Growth, Maturation, and Fertilization in Vitro. Biology of Reproduction, 41, 268-276. https://doi.org/10.1095/biolreprod41.2.268

[11] Eppig, J.J. and O’Brien, M.J. (1996) Development in Vitro of Mouse Oocytes from Primordial Follicles. Biology of Reproduction, 54, 197-207.

https://doi.org/10.1095/biolreprod54.1.197

[12] Edmondson, R., Broglie, J.J., Adcock, A.F. and Yang, L. (2014) Three-Dimensional Cell Culture Systems and Their Applications in Drug Discovery and Cell-Based Biosensors. Assay and Drug Development Technologies, 12, 207-218. https://doi.org/10.1089/adt.2014.573

[13] O’Brien, M.J., Pendola, J.K. and Eppig, J.J. (2003) A Revised Protocol for In Vitro Development of Mouse Oocytes from Primordial Follicles Dramatically Improves Their Developmental Competence. Biology of Reproduction, 68, 1682-1686. https://doi.org/10.1095/biolreprod.102.013029

[14] Spears, N., De Bruin, J. and Gosden, R. (1996) The Establishment of Follicular Dominance in Co-Cultured Mouse Ovarian Follicles. Journal of Reproduction and Fertility, 106, 1-6. https://doi.org/10.1530/jrf.0.1060001

[15] Buccione, R., Cecconi, S., Tatone, C., Mangia, F. and Colonna, R. (1987) Follicle Cell Regulation of Mammalian Oocyte Growth. Journal of Experimental Zoology, 242, 351-354. https://doi.org/10.1002/jez.1402420314

[16] Eppig, J.J. (1977) Mouse Oocyte Development in Vitro with Various Culture Systems. Developmental Biology, 60, 371-388. https://doi.org/10.1016/0012-1606(77)90135-X

[17] Eppig, J.J. (1979) A Comparison between Oocyte Growth in Coculture with Granulosa Cells and Oocytes with Granulosa Cell-Oocyte Junctional Contact Maintained in Vitro. Journal of Experimental Zoology, 209, 345-353.

https://doi.org/10.1002/jez.1402090216

[18] Gomes, J.E., Correia, S.C., Gouveia-Oliveira, A., Cidadao, A.J. and Plancha, C.E. (1999) Three-Dimensional Environments Preserve Extracellular Matrix Compartments of Ovarian Follicles and Increase FSH-Dependent Growth. Molecular Reproduction and Development, 54, 163-172. https://doi.org/10.1002/(SICI)1098-2795(199910)54:2<163::AID-MRD8>3.0.CO;2-4 
[19] Gook, D.A., Edgar, D., Lewis, K., Sheedy, J. and Gardner, D. (2013) Impact of Oxygen Concentration on Adult Murine Pre-Antral Follicle Development in Vitro and the Corresponding Metabolic Profile. Molecular Human Reproduction, 20, 31-41. https://doi.org/10.1093/molehr/gat062

[20] Kreeger, P.K., Deck, J.W., Woodruff, T.K. and Shea, L.D. (2006) The in Vitro Regulation of Ovarian Follicle Development Using Alginate-Extracellular Matrix Gels. Biomaterials, 27, 714-723. https://doi.org/10.1016/j.biomaterials.2005.06.016

[21] Lundy, T., Smith, P., O’Connell, A., Hudson, N.L. and McNatty, K.P. (1999) Populations of Granulosa Cells in Small Follicles of the Sheep Ovary. Journal of Reproduction and Fertility, 115, 251-262. https://doi.org/10.1530/jrf.0.1150251

[22] Merdassi, G., Mazoyer, C., Guerin, J.F., Saad, A., Salle, B. and Lornage, J. (2011) Examination of Viability and Quality of Ovarian Tissue after Cryopreservation Using Simple Laboratory Methods in Ewe. Reproductive Biology and Endocrinology, 9, 78. https://doi.org/10.1186/1477-7827-9-78

[23] Aerts, J., Martinez-Madrid, B., Flothmann, K., De Clercq, J., Van Aelst, S. and Bols, P. (2008) Quantification and Viability Assessment of Isolated Bovine Primordial and Primary Ovarian Follicles Retrieved through a Standardized Biopsy Pick-Up Procedure. Reproduction in Domestic Animals, 43, 360-366.

https://doi.org/10.1111/j.1439-0531.2007.00915.x

[24] Campos, J.R., Rosa-e-Silva, J.C., Carvalho, B.R., Vireque, A.A., Silva-de-Sá, M.F. and Rosa-e-Silva, A.C.J. (2011) Cryopreservation Time Does Not Decrease Follicular Viability in Ovarian Tissue Frozen for Fertility Preservation. Clinics, 66, 2093-2097. https://doi.org/10.1590/S1807-59322011001200015

[25] Li, J., Gao, H., Tian, Z., Wu, Y., Wang, Y., Fang, Y., Lin, L., Han, Y., Wu, S. and Haq, I. (2016) Effects of Chronic Heat Stress on Granulosa Cell Apoptosis and Follicular Atresia in Mouse Ovary. Journal of Animal Science and Biotechnology, 7, 57. https://doi.org/10.1186/s40104-016-0116-6

[26] Lunardi, F.O., Araújo, V.R., Faustino, L.R., de Andrade Carvalho, A., Gonçalves, R.F.B., Bass, C.S., Báo, S.N., Name, K.P.O., Campello, C.C. and de Figueiredo, J.R. (2012) Morphologic, Viability and Ultrastructural Analysis of Vitrified Sheep Preantral Follicles Enclosed in Ovarian Tissue. Small Ruminant Research, 107, 121-130. https://doi.org/10.1016/j.smallrumres.2012.04.009

[27] Santos, R.R., Tharasanit, T., Figueiredo, J.R., Van Haeften, T. and Van den Hurk, R. (2006) Preservation of Caprine Preantral Follicle Viability after Cryopreservation in Sucrose and Ethylene Glycol. Cell and Tissue Research, 325, 523-531.

https://doi.org/10.1007/s00441-006-0193-5

[28] Santos, R.R., Van Haeften, T., Roelen, B.A., Knijn, H.M., Colenbrander, B., Gadella, B.M. and Van den Hurk, R. (2008)Osmotic Tolerance and Freezability of Isolated Caprine Early-Staged Follicles. Cell and Tissue Research, 333, 323-331. https://doi.org/10.1007/s00441-008-0613-9

[29] Irving-Rodgers, H.F., Hummitzsch, K., Murdiyarso, L.S., Bonner, W.M., Sado, Y., Ninomiya, Y., Couchman, J.R., Sorokin, L.M. and Rodgers, R.J. (2010) Dynamics of Extracellular Matrix in Ovarian Follicles and Corpora Lutea of Mice. Cell and Tissue Research, 339, 613-624. https://doi.org/10.1007/s00441-009-0905-8

[30] Rodgers, R., Van Wezel, I., Irving-Rodgers, H., Lavranos, T., Irvine, C. and Krupa, M. (1998) Roles of Extracellular Matrix in Follicular Development. Journal of Reproduction and Fertility Supplement, 54, 343-352.

[31] Rodgers, R.J., Irving-Rodgers, H.F. and Russell, D.L. (2003) Extracellular Matrix of the Developing Ovarian Follicle. Reproduction, 126, 415-424. 
https://doi.org/10.1530/rep.0.1260415

[32] van Wezel, I.L. and Rodgers, R.J. (1996) Morphological Characterization of Bovine Primordial Follicles and Their Environment in Vivo. Biology of Reproduction, 55, 1003-1011. https://doi.org/10.1095/biolreprod55.5.1003

[33] Salani, R., Neuberger, I., Kurman, R.J., Bristow, R.E., Chang, H.-W., Wang, T.-L. and Shih, I.-M. (2007) Expression of Extracellular Matrix Proteins in Ovarian Serous Tumors. International Journal of Gynecological Pathology, 26, 141-146. https://doi.org/10.1097/01.pgp.0000229994.02815.f9

[34] Salvetti, N.R., Gimeno, E.J., Canal, A.M., Lorente, J.A. and Ortega, H.H. (2003) Histochemical Study of the Extracellular Matrix Components in the Follicular Wall of Induced Polycystic Ovaries. Brazilian Journal of Morphological Sciences, 20, 93-100.

[35] Xu, M., West, E., Shea, L.D. and Woodruff, T.K. (2006) Identification of a Stage-Specific Permissive in Vitro Culture Environment for Follicle Growth and Oocyte Development. Biology of Reproduction, 75, 916-923.

https://doi.org/10.1095/biolreprod.106.054833

[36] Parrish, E.M., Siletz, A., Xu, M., Woodruff, T.K. and Shea, L.D. (2011) Gene Expression in Mouse Ovarian Follicle Development in Vivo versus an ex Vivo Alginate Culture System. Reproduction, 142, 309-318.

https://doi.org/10.1530/REP-10-0481

[37] Xu, J., Lawson, M., Yeoman, R., Pau, K., Barrett, S., Zelinski, M. and Stouffer, R. (2011) Secondary Follicle Growth and Oocyte Maturation during Encapsulated Three-Dimensional Culture in Rhesus Monkeys: Effects of Gonadotrophins, Oxygen and Fetuin. Human Reproduction, 26, 1061-1072.

https://doi.org/10.1093/humrep/der049

[38] Kreeger, P.K., Fernandes, N.N., Woodruff, T.K. and Shea, L.D. (2005) Regulation of Mouse Follicle Development by Follicle-Stimulating Hormone in a ThreeDimensional in Vitro Culture System Is Dependent on Follicle Stage and Dose. Biology of Reproduction, 73, 942-950. https://doi.org/10.1095/biolreprod.105.042390

[39] Kreeger, P.K., Woodruff, T.K. and Shea, L.D. (2003) Murine Granulosa Cell Morphology and Function Are Regulated by a Synthetic Arg-Gly-Asp Matrix. Molecular and Cellular Endocrinology, 205, 1-10.

[40] Shikanov, A., Xu, M., Woodruff, T.K. and Shea, L.D. (2011) A Method for Ovarian Follicle Encapsulation and Culture in a Proteolytically Degradable 3 Dimensional System. JoVE (Journal of Visualized Experiments), 15, e2695.

[41] Oktem, O. and Oktay, K. (2007) The Role of Extracellular Matrix and Activin-A in in Vitro Growth and Survival of Murine Preantral Follicles. Reproductive Sciences, 14, 358-366. https://doi.org/10.1177/1933719107303397

[42] Lee, J., Cuddihy, M.J. and Kotov, N.A. (2008) Three-Dimensional Cell Culture Matrices: State of the Art. Tissue Engineering Part B: Reviews, 14, 61-86.

https://doi.org/10.1089/teb.2007.0150

[43] Cui, X., Dini, S., Dai, S., Bi, J., Binder, B., Green, J. and Zhang, H. (2016) A Mechanistic Study on Tumour Spheroid Formation in Thermosensitive Hydrogels: Experiments and Mathematical Modelling. RSC Advances, 6, 73282-73291. https://doi.org/10.1039/C6RA11699J

[44] Griffin, J., Emery, B.R., Huang, I., Peterson, C.M. and Carrell, D.T. (2006) Comparative Analysis of Follicle Morphology and Oocyte Diameter in Four Mammalian Species (Mouse, Hamster, Pig, and Human). Journal of Experimental \& Clinical As- 
sisted Reproduction, 3, 2. https://doi.org/10.1186/1743-1050-3-2

[45] Young, J. and McNeilly, A.S. (2010) Theca: The Forgotten Cell of the Ovarian Follicle. Reproduction, 140, 489-504. https://doi.org/10.1530/REP-10-0094

[46] Young, F., Drummond, J., Akers, E., Bartle, L., Kennedy, D. and Asaduzzaman, M. (2017) Effects of Ovarian Disaggregation on Adult Murine Follicle Yield and Viability. Reproduction, Fertility and Development, 29, 2400-2410. https://doi.org/10.1071/RD16398

[47] Dolmans, M.-M., Michaux, N., Camboni, A., Martinez-Madrid, B., Van Langendonckt, A., Nottola, S.A. and Donnez, J. (2006) Evaluation of Liberase, a Purified Enzyme Blend, for the Isolation of Human Primordial and Primary Ovarian Follicles. Human Reproduction, 21, 413-420. https://doi.org/10.1093/humrep/dei320

[48] Torrance, C., Telfer, E. and Gosden, R. (1989) Quantitative Study of the Development of Isolated Mouse Pre-Antral Follicles in Collagen Gel Culture. Journal of Reproduction and Fertility, 87, 367-374. https://doi.org/10.1530/jrf.0.0870367

[49] Telfer, E. (1996) The Development of Methods for Isolation and Culture of Preantral Follicles from Bovine and Porcine Ovaries. Theriogenology, 45, 101-110. https://doi.org/10.1016/0093-691X(95)00359-G

[50] Lintern-Moore, S. and Moore, G. (1979) The Initiation of Follicle and Oocyte Growth in the Mouse Ovary. Biology of Reproduction, 20, 773-778. https://doi.org/10.1095/biolreprod20.4.773

[51] Pedersen, T. and Peters, H. (1968) Proposal for a Classification of Oocytes and Follicles in the Mouse Ovary. Journal of Reproduction and Fertility, 17, 555-557. https://doi.org/10.1530/jrf.0.0170555

[52] Stojkovic, M., Machado, S.A., Stojkovic, P., Zakhartchenko, V., Hutzler, P., Gonçalves, P.B. and Wolf, E. (2001) Mitochondrial Distribution and Adenosine Triphosphate Content of Bovine Oocytes before and after in Vitro Maturation: Correlation with Morphological Criteria and Developmental Capacity after in Vitro Fertilization and Culture. Biology of reproduction, 64, 904-909. https://doi.org/10.1095/biolreprod64.3.904

[53] Orimoto, A.M., Dumaresq-Doiron, K., Jiang, J.-Y., Tanphaichitr, N., Tsang, B.K. and Carmona, E. (2008) Mammalian Hyaluronidase Induces Ovarian Granulosa cell Apoptosis and Is Involved in Follicular Atresia. Endocrinology, 149, 5835-5847. https://doi.org/10.1210/en.2008-0175

[54] Devine, P.J., Perreault, S.D. and Luderer, U. (2012) Roles of Reactive Oxygen Species and Antioxidants in Ovarian Toxicity. Biology of Reproduction, 86, 27. https://doi.org/10.1095/biolreprod.111.095224

[55] Xian, J.-A., Wang, A.-L., Miao, Y.-T. and Li, B. (2013) Flow Cytometric Analysis of in Vitro Cytotoxicity of Cadmium in Haemocytes from the Tiger Shrimp, Penaeus Monodon. Bulletin of Environmental Contamination and Toxicology, 90, 46-50. https://doi.org/10.1007/s00128-012-0839-9

[56] Filatov, M., Khramova, Y. and Semenova, M. (2015) In Vitro Mouse Ovarian Follicle Growth and Maturation in Alginate Hydrogel: Current State of the Art. Acta Naturae, 7, 48-56.

[57] Silva, C., Matos, M., Rodrigues, G., Faustino, L., Pinto, L., Chaves, R., Araújo, V., Campello, C. and Figueiredo, J. (2010) In Vitro Survival and Development of Goat Preantral Follicles in Two Different Oxygen Tensions. Animal Reproduction Science, 117, 83-89. https://doi.org/10.1016/j.anireprosci.2009.03.015

[58] Heise, M.K., Koepsel, R., McGee, E.A., Russell and A.J. (2009) Dynamic Oxygen 
Enhances Oocyte Maturation in Long-Term Follicle Culture. Tissue Engineering Part C: Methods, 15, 323-332. https://doi.org/10.1089/ten.tec.2007.0418

[59] Baarends, W.M., Uilenbroek, J., Kramer, P., Hoogerbrugge, J.W., Van Leeuwen, E., Themmen, A. and Grootegoed, J.A. (1995) Anti-Müllerian Hormone and Anti-Müllerian Hormone Type II Receptor Messenger Ribonucleic Acid Expression in Rat Ovaries during Postnatal Development, the Estrous Cycle, and Gonadotropin-Induced Follicle Growth. Endocrinology, 136, 4951-4962. https://doi.org/10.1210/endo.136.11.7588229

[60] Pask, A.J., Whitworth, D.J., Mao, C.-A., Wei, K.-J., Sankovic, N., Graves, J.A., Shaw, G., Renfree, M.B. and Behringer, R.R. (2004) Marsupial Anti-Müllerian Hormone Gene Structure, Regulatory Elements, and Expression. Biology of Reproduction, 70, 160-167. https://doi.org/10.1095/biolreprod.103.020016

[61] Webber, L., Stubbs, S., Stark, J., Trew, G., Margara, R., Hardy, K. and Franks, S. (2003) Formation and Early Development of Follicles in the Polycystic Ovary. The Lancet, 362, 1017-1021. https://doi.org/10.1016/S0140-6736(03)14410-8

[62] Weenen, C., Laven, J.S., von Bergh, A.R., Cranfield, M., Groome, N.P., Visser, J.A., Kramer, P., Fauser, B.C. and Themmen, A.P. (2004) Anti-Müllerian Hormone Expression Pattern in the Human Ovary: Potential Implications for Initial and Cyclic Follicle Recruitment. Molecular Human Reproduction, 10, 77-83.

https://doi.org/10.1093/molehr/gah015 